\title{
Study of mechanochemical interaction of tungsten with liquid gallium
}

\author{
Vladimir M. Grigoryev ${ }^{*}$, Sergey V. Vosmerikov ${ }^{2}$ \\ ${ }^{1}$ Novosibirsk State Technical University, 630073, Novosibirsk, Russia \\ ${ }^{2}$ Institute of Solid State Chemistry and Mechanochemistry SB RAS, 630128, Novosibirsk, Russia
}

\begin{abstract}
Mechanochemical interaction of tungsten with gallium was studied. Using x-ray diffraction and electron microscopy, it was shown that after mechanical activation of a W-Ga mixture for $1 \mathrm{~min}$, gallium is completely spread, as a thin layer, onto the tungsten surface, while the longer activation leads to the formation of $\mathrm{W}(\mathrm{Ga})$ solid solution. Moreover, gallium was shown to be incorporated into tungsten lattice only if the grains reach a certain critical nanosize.
\end{abstract}

It is well known that the rate of heterogeneous reaction depends on the value of the contact surface. The contact is point-like for a solid phase component, so the total contact surface is small, and the process is long-lasting. It leads to significant contamination of the product with the material of milling bodies and products of mechanochemical interaction of the mixture with the atmosphere of the synthesis that limits the use of the method considerably. The most effective way to minimize this limitation is to decrease the time of mechanical activation (MA) of reagents notably, which, for instance, is possible by increasing the rate of the chemical reactions.

One of the most effective ways to increase the rate of interaction in heterophase systems is to increase the phase contact area of the initial reagents with the same quantity of the dispersed phase. The largest value of the contact surface can be achieved if one of the components is liquid. In that case, the contact surface becomes the total surface area of the solid phase as long as it is fully soaked in the liquid.

Good wetting of the solid metal by liquid one ensures a large contact surface between the reagents. Contact surface increases by several orders with the second component being in a liquid state in relation to solid-phase interaction. That is why these systems are expected to have extremely high rates of mechanochemical interaction and other distinct qualities different from solid-phase synthesis.

Analysis of literature signifies that researches about mechanochemical synthesis in solid-liquid metal systems have not been paid enough attention, especially systems with low heat of mixing.

This study is dedicated to the research of mechanochemical interaction in the W-Ga system, in which heat of mixing is $1 \mathrm{~kJ} / \mathrm{mol}$, according to the calculation using Miedema's model.

*Corresponding author: 3 ebc98@mail.ru 
W-Ga system is not well studied, stability diagram for it has not been developed. There is information about a very insignificant solubility [1] and about the appearance of two metastable intermetallics $\mathrm{Ga}_{5} \mathrm{~W}_{2}$ and $\mathrm{Ga}_{3} \mathrm{~W}_{3}$ under high pressure, that under normal conditions break down into elements $[2,3]$ and about particularly weak corrosion of tungsten in gallium, which is equal to $10^{-6} \mathrm{~g} / \mathrm{m}^{2} \cdot \mathrm{h}$ at $1173-1473 \mathrm{~K}$ (for comparison, the corrosion of copper in gallium is $1000 \mathrm{~g} / \mathrm{m}^{2} \cdot \mathrm{h}$ at $373-573 \mathrm{~K}$ ).

The research of the interaction of tungsten with liquid gallium with and without mechanical activation was conducted. 20 at.\% liquid gallium spreads on the surface of the micrometric tungsten powder in a thin layer, undetectable by diffraction, after several minutes (Fig. 1A), at the same time 40at.\% does not spread fully (Fig. 1B). However, mechanical activation of $\mathrm{W}+40$ at. $\%$ Ga mixture during even 1 min leads to full spreading of gallium on the tungsten surface, on XRD patterns only tungsten reflections are recorded (Fig. 2A), upon that diffraction reflection of gallium disappear without a noticeable broadening of tungsten reflections.

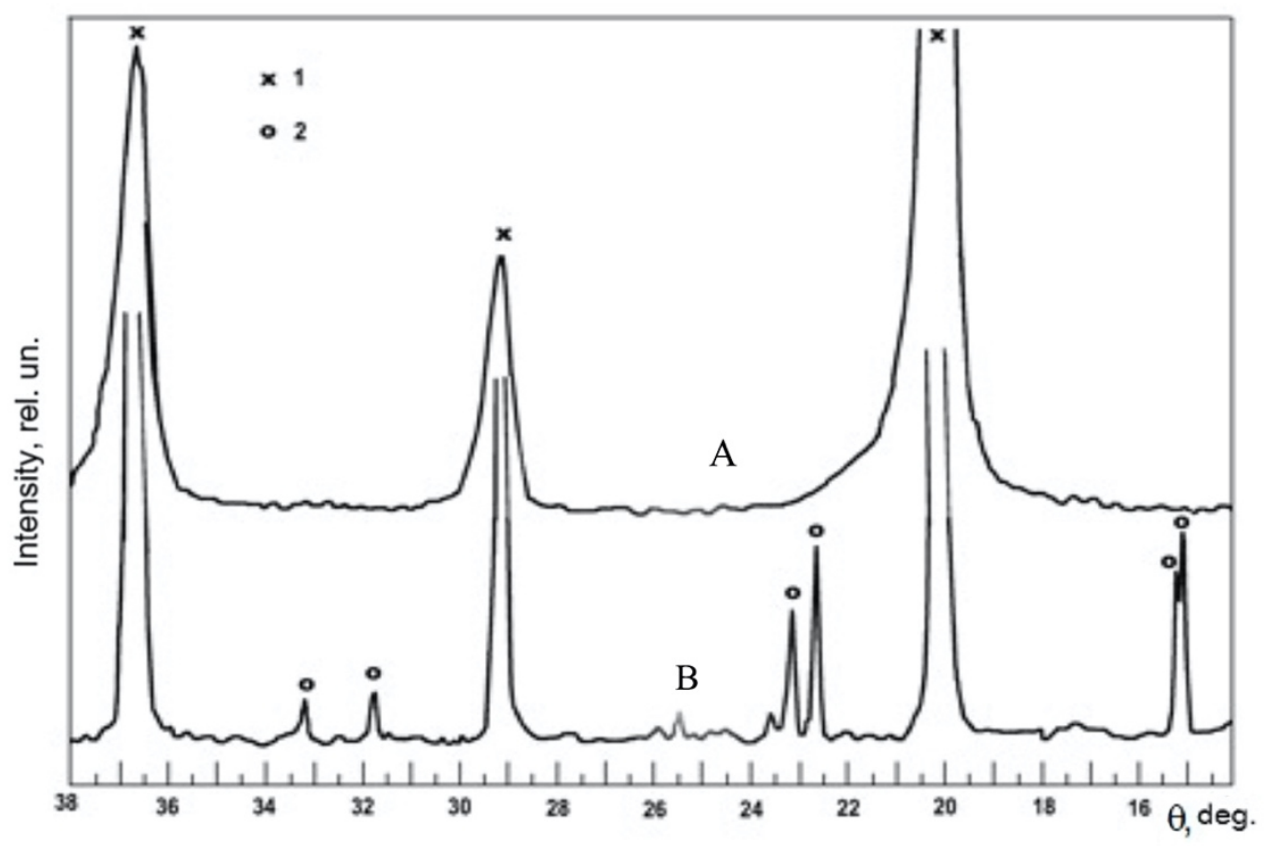

Fig. 1. X-ray diffraction patterns of the $\mathrm{W}+20$ at. $\%$ Ga mixture (A) and $\mathrm{W}+40$ at.\% Ga mixture (B) after exposure at $37^{\circ} \mathrm{C}$ for $5 \mathrm{~h} .1-\mathrm{W}, 2-\mathrm{Ga}$. 


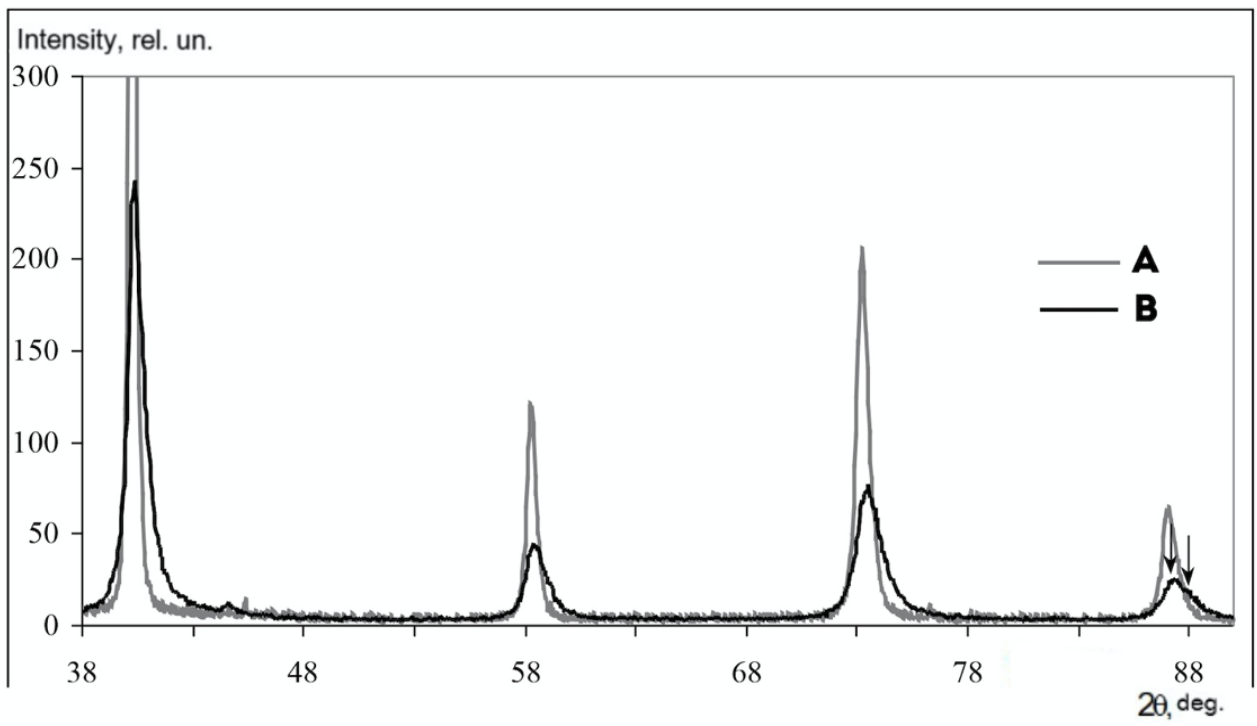

Fig. 2. $\mathrm{X}$-ray diffraction patterns of $\mathrm{W}+20$ at. $\%$ Ga mixture after 1 (A) and $20 \mathrm{~min}$ (B) MA

The absence of gallium diffraction reflections suggests that it spread on the surface of tungsten particles in a particularly thin layer and did not have its crystalline structure because according to the information from chemical analysis, gallium is present in the system in the initial quantity.

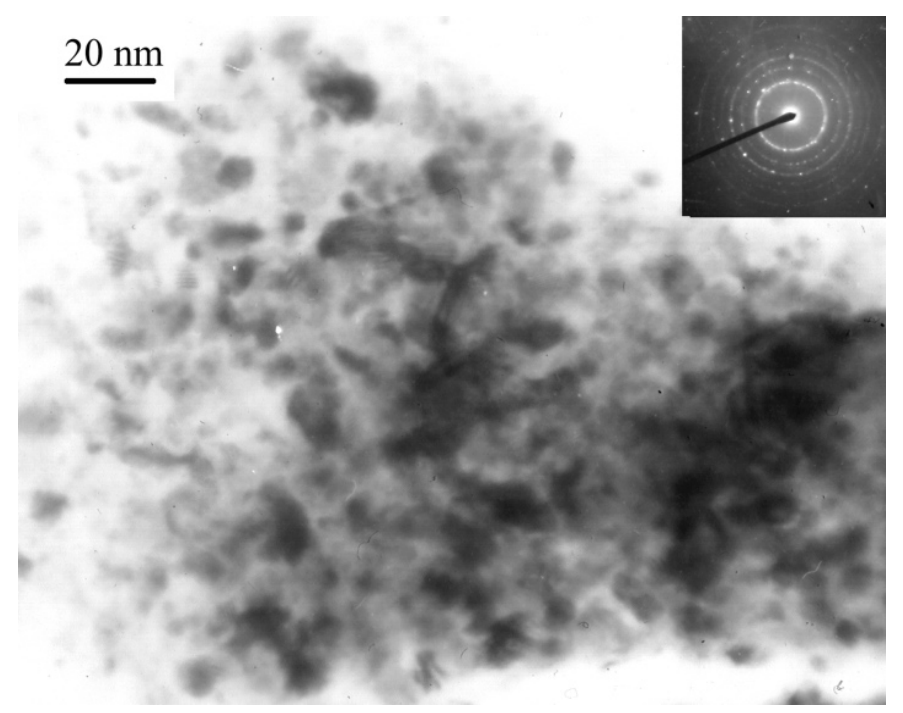

Fig. 3. Micrograph of the high resolution of $\mathrm{W}+20 \mathrm{wt} . \%$ Ga mixture after activation for $20 \mathrm{~min}$

Further activation of these composites leads to the significant decrease of the intensity and broadening of tungsten reflections (Fig. 2B), as well as to the splitting of the diffraction reflection (220). The forming phase size is nanometric (Fig. 3).

Comparing the XRD patterns of $\mathrm{W}+\mathrm{Ga}$ samples after activation for 1 and 20 minutes it is clear that during the long-term activation there is a visible asymmetry in tungsten reflections, which shows that solid solution $\mathrm{W}(\mathrm{Ga})$ begins to form (Fig. 2). 
Therefore, the conducted researches suggest that during the mechanical interaction between a solid and a liquid metal there is spreading on the solid surface, particularly on the one, newly formed during the dispersion, obtaining the composite, in which a thin layer of liquid metal covers the nanosized solid metal. According to electron microscopy and $\mathrm{x}$-ray diffraction, it is possible to say that insertion of gallium into the tungsten lattice with the formation of a solid solution happens only after reaching a certain nanosize of the grains (on average the size of the solid phase component is $\sim 10 \mathrm{~nm}$ ).

The work was carried out within the state assignment of the Institute of Solid State Chemistry and Mechanochemistry SB RAS (No. 121032500062-4).

\section{References}

1. S.P. Yatsenko, Gallium (Nauka, Moscow, 1974)

2. S.V. Popova, L.N. Fomicheva, J. Less Common Met. 77, 137 (1981)

3. S.V. Popova, Phys. Scr. 1, 131 (1982) 